\title{
Anaplastic lymphoma kinase tyrosine kinase inhibitors in non-small cell lung cancer
}

\author{
Tiziana Vavalà ${ }^{1}$, Annapaola Mariniello², Silvia Novelloº \\ ${ }^{1}$ SC of Oncology, ASL CN1, Ospedale Civile di Saluzzo, Saluzzo, CN, Italy; ${ }^{2}$ Department of Oncology, University of Torino, AOU S. Luigi \\ Gonzaga, Orbassano, TO, Italy \\ Contributions: (I) Conception and design: All Authors; (II) Administrative support: None; (III) Provision of study materials or patients: None; (IV) \\ Collection and assembly of data: All Authors; (V) Data analysis and interpretation: All Authors; (VI) Manuscript writing: All authors; (VII) Final \\ approval of manuscript: All authors. \\ Correspondence to: Tiziana Vavalà, MD. SC of Oncology at ASL CN1, Ospedale Civile di Saluzzo, via Spielberg 37, 12037 Saluzzo, CN, Italy. \\ Email: tvavala@hotmail.it; tvavala@gmail.com.
}

\begin{abstract}
Lung cancer still represents the leading cause of cancer-related mortality. However, the recent advent of tyrosine kinase inhibitors (TKI), pioneering drugs against targetable mutations, have dramatically improved prognosis of advanced non-small cell lung cancer (NSCLC) patients. Anaplastic lymphoma kinase $(A L K)$ gene rearrangements, identified in $3-7 \%$ of NSCLC cases, reflects in the constitutive activation of downstream signalling pathways, stimulating tumour cell proliferation, differentiation and survival. To accurately detect the wide spectrum of ALK rearrangements, the introduction of innovative techniques, like reverse transcriptase polymerase chain reaction (RT-PCR) or next generation sequencing (NGS) now allows for a more precise detection of variants and a more objective reading assessment, compared to the traditional diagnostic approaches. In some occasions, these new tools may dynamically monitor tumor evolution and even guide the choice of the most appropriate ALK inhibitor. In fact, among ALK TKIs available, crizotinib was the first to receive FDA accelerate approval for ALK rearranged NSCLC patients. Notwithstanding its response rate, ranging from $57 \%$ to $74 \%$, the majority of patients progress within the first year of drug administration, due to acquired resistance. Both ALK-dependent and independent mechanisms of acquired resistance to TKIs have been identified. If the activation of multiple bypass signaling pathways constitutes the most common ALK-independent mechanism of resistance and one of the most difficult to overcome, ALK-dependent escape strategy mainly consists of mutations in the kinase domain, where the type of mutation largely depends on the TKI administered. Second and third generation TKIs are now available and are demonstrating high systemic and central nervous system (CNS) efficacy in clinical trials. Even though appropriate timing and sequencing of these compounds are still unclear, the large number of ALK inhibitors is now a precious resource aiming to prolong progression-free survival (PFS) and finally overall survival (OS). Here Authors provide an overview of the current approaches in the clinical management of advanced NSCLC patients harboring ALK rearrangement and discuss future perspectives to address current issues, highlighting the perception that ALK-rearranged advanced NSCLC patients benefit from maintained ALK inhibition for as long as possible.
\end{abstract}

Keywords: Non-small cell lung cancer (NSCLC); anaplastic lymphoma kinase (ALK); ALK inhibitors; ALK tyrosine kinase inhibitor (ALK-TKI)

Submitted Sep 15, 2018. Accepted for publication Oct 08, 2018.

doi: $10.21037 /$ tcr.2018.10.23

View this article at: http://dx.doi.org/10.21037/tcr.2018.10.23 


\section{Introduction and molecular pathology of anaplastic lymphoma kinase (ALK)-rearranged non-small cell lung cancer (NSCLC)}

Lung cancer is diagnosed in a large number of cases and, with 1.69 million deaths detected in 2015, it is the foremost cause of cancer-related death (1). Recently, pioneering drugs against molecular targets, primarily for patients with advanced NSCLC expressing epidermal growth factor receptor (EGFR) mutations, subsequently for those who presented ALK rearrangements, have considerably improved their prognosis (2).

$A L K$ gene is placed on chromosome 2, it encodes for a trans-membrane tyrosine kinase receptor, the ALK, member of the insulin receptor super-family. $A L K$ gene rearrangements were identified in $3-7 \%$ of NSCLC cases: the short-arm inversion (inv 2-p21p23) in chromosome 2 produces a fusion of echinoderm microtubule-associated protein-like 4 (EML4) gene exons 1-13 with exons 20-29 of $A L K$ gene (2); this resulted EML4-ALK kinase induces a dimerization of ALK receptor in a ligand-independent manner, and a consequent constitutive activation of downstream signalling pathways as JAK/STAT (Janus kinase/signal transducer and activator of transcription), PI3K/AKT (phosphatidylinositol-3 kinase/protein kinase B) and MAPK/ERK (mitogen-activated kinaselike protein/extracellular-signal regulated kinase) ones, inducing cell proliferation, differentiation and survival (3). Rearrangement of EML4-ALK is more frequently expressed in younger patients, non-smokers, in signet-ring cell adenocarcinomas of Caucasian patients and acinus forms of adenocarcinomas of Asian ones. The alteration usually occurs independently of KRAS (Kirsten-rat-sarcoma) or EGFR mutations and, even within a very small amount of cases, it is not mutually exclusive, meaning that subjects harbouring EGFR mutations should not be ruled out from ALK baseline evaluation. Ultimately and differently from EGFR mutations, EML4-ALK rearrangement presents comparable incidence worldwide (4). ALK intracellular tyrosine kinase domain, fusing with other EML4 truncations, produces also variants which include the entire ALK intracellular domain, codified by exons 20 to 29, but differ in EML4 gene point of fusion (4). However, EML4 is not an exclusive fusion partner with ALK as different even if unusual companions like KIF5B (kinesin family member 5B), TFG (TRK-fused gene), or KLC1 (kinesin light chain 1), were also evidenced (5).

For an accurate detection of ALK rearrangements, in clinical practice, the association of a fluorescent in situ hybridization (FISH) technique with immunohistochemistry (IHC) is currently recommended and can be performed on either histological or cytological specimens (5). FISH test was the first procedure to be described as the gold standard and the companion diagnostic in FDA crizotinib approval. With the advent of anti-ALK antibodies, IHC demonstrated to be a viable option, also in those cases with small tissue samples, with the advantage, compared to FISH, to be reproducible, cost and time effective. The most extensively used antibodies are D5F3 (Cell Signaling Technology, Danvers, MA, USA) and 54A (Novocastra, Leica Biosystems, Buffalo Grove, IL, USA) clones (6). Comparatives analyses were carried out for IHC, especially with $\mathrm{D} 5 \mathrm{~F} 3$ clone, and FISH, reporting high concordance (sensitivity ranged from $81 \%$ to $100 \%$ and specificity from $82 \%$ to $100 \%$, respectively), according to the different studies considered (7). In any case, the diagnostic tools just mentioned have several limitations: FISH test results could be affected by inappropriate sample fixation or false negative results could be evidenced in those cases of complex ALK rearrangements. Similarly, IHC pitfalls could often be due to misinterpretation in labelling reading (influenced by necrotic areas, macrophages in tumor specimen or delay in fixation) with the risk of false positive results. Moreover, important limitations are common to both the approaches and lie in tumor heterogeneity, subjectivity in result assessments and inability to detect uncommon variants (7).

Innovative techniques like reverse transcriptase polymerase chain reaction (RT-PCR) or next generation sequencing (NGS) were then improved and now allow to face these issues, at least in part: multiplexed RT-PCR consents to identify pre-defined ALK fusion gene variants but the clinical utility of this tool has been questioned due to the high costs, expertise needed, bias derived by the quality and quantity of extracted RNA and finally the suboptimal performance on formalin-fixed paraffin-embedded tissue sections (8). NGS rapidly detects all spectrum of ALK rearrangements and simultaneously identifies further gene mutations/translocations that may interfere with treatment choices (e.g., ROS-1, c-ROS oncogene 1, RET rearranged during transfection, MET proto-oncogene). Tumor sequencing can also clarify discordant results between IHC and FISH, more common in cases of borderline FISH positivity (15-20\%) (7). An additional advantage is NGS application on blood samples (liquid biopsy), with the aim to partially solve tumor heterogeneity issues while dynamically monitoring tumor evolution, particularly along 
ALK inhibitors administration (9).

\section{Options of treatment and acquired resistance for advanced ALK-rearranged NSCLC patients}

Treatment of ALK-dependent NSCLC typically starts with an ALK tyrosine kinase inhibitor (TKI). Crizotinib is a multi-target MET, ALK and ROS1 TKI; its efficacy was described in PROFILE 1014, a front-line phase III trial which showed a 10.9 versus 7 months [hazard ratio (HR): 0.45 , $95 \%$ confidence interval $(\mathrm{CI}), 0.35-0.60 \mathrm{P}<0.0001]$ benefit in progression-free survival (PFS) and a $74 \%$ versus $45 \%$ objective response rate (ORR) compared to standard first-line platinum/pemetrexed chemotherapy in patients with ALKrearranged NSCLC, receiving, for this reason, accelerate approval from US Food and Drug Administration (FDA) (10).

However, even if crizotinib, as first-generation ALK inhibitor, presents an ORR ranging from $57 \%$ to $74 \%$, the majority of patients progress within the first 12 months, with 11.3 months as a median duration of response. In this context two major mechanisms of acquired resistance to ALK TKIs were identified: (I) ALK-dependent: where cell addiction from ALK signalling endures even with presence of ALK amplification or resistance mutations; and (II) ALKindependent: such as by-pass signaling, drug efflux pumps or phenotypic changes like epithelial-to-mesenchymal transition (11). Primary resistance mechanisms were also documented, but for now they are poorly understood and currently under evaluation (11). Regarding ALK-dependent acquired resistance, it mainly consists of mutations in the kinase domain, whilst $A L K$ gene amplification is a pretty uncommon event in patients progressing to crizotinib. In contrast with EGFR gatekeeper mutation T790M, in ALK rearranged NSCLC patients the type of mutation largely depends on the TKI administered, particularly in crizotinib-resistant patients usual alterations are G1269A or L1196M (whereas the first engages the crizotinib binding site, the latter affects the catalytic domain in the ATP pocket), G1202R ALK mutation is present in around $2 \%$ of patients progressing to crizotinib but it induces resistance also to next-generation ALK TKIs (ceritinib, alectinib and brigatinib) while it is proficiently inhibited by lorlatinib, a third-generation inhibitor (11). Wild-type EML4-ALK amplifications or those of ALK fusion gene (about 13\%) induce acquired drug resistance independently from concurrent ALK mutations (e.g., concomitant ALK CNG and G1269A mutations reported in one patient) (12).
Among ALK-independent mechanisms of resistance, creation of multiple pathways for by-passing the signal is the most common escape strategy and represents one of the most difficult to overcome. Pathways involved include KRAS mutations and EGFR, RAS/MEK (rat-sarcoma/ mitogen-activated protein-kinase), HSP90 (heat-shockprotein-90) or PI3K/AKT/mTOR (PI3K/AKT/mammalian target of rapamycin) pathways, phospho-EGFR, phosphoALK, phospho-HER3 (human epidermal growth factor receptor-3) or phospho-IGFR-1R (insulin-like growth factor-1 receptor) overexpressions, and amplifications of KIT (KIT proto-oncogene receptor tyrosine kinase). Multidrug resistance 1 ( $M D R 1)$ gene encodes for $\mathrm{P}$-glycoprotein (P-gp), a highly-conserved ATP-dependent efflux pump which is a mechanism of resistance often responsible for central nervous system (CNS) disease progression. Finally, epithelial-to-mesenchymal transition represents an acquired resistance mechanism also described in patients receiving ceritinib, but still under evaluation (12).

In this context, it is important to highlight an interesting, innovative molecular concern: dissimilar EML4-ALK fusion variants predict different responses and/or disease control to ALK inhibitor crizotinib; this event is due to protein stability from EML4-ALK variants which influences the overall fusion stability, even also protein degradation induced by the inhibitor used and its consequent drug sensitivity. Particularly, it was evidenced that EML4-ALK variant 1 induces a similar ORR to crizotinib ( $74 \%$ versus $63 \%$ ) but a higher disease control rate (DCR, 95\% versus 63\%) and a prolonged PFS (11 versus 4.2 months) than other variants (13). Woo et al. evidenced, for variants $1 / 2$ /others group, a 2-year PFS of $69 \%$ (95\% CI, 49.9-95.4\%) versus $32.7 \%$ (95\% CI, $15.6-68.4 \%$ ) of group variants $3 \mathrm{a} / \mathrm{b}$ $(\mathrm{P}=0.10)$ among crizotinib-, ceritinib- and alectinib-treated patients. Cells with variant $3 \mathrm{a}$ or $5 \mathrm{a}$ were refractory to ALK TKI with $>10$-fold higher half maximal inhibitory concentration in vitro (14).

Considering previous reasons, next-generation ALK inhibitors development was enhanced and ceritinib (LDK378), alectinib (CH5424802) and brigatinib (AP26113) obtained FDA approval for treatment of ALKrearranged NSCLC patients refractory to crizotinib; subsequently lorlatinib (PF-06463922) received a breakthrough-therapy designation in the same setting. Innovative ALK TKIs as ensartinib (X-396) or entrectinib (RXDX-101) are presently under clinical evaluation (15) (Table 1). 
Table 1 Ongoing trials with ALK TKIs as monotherapy in NSCLC

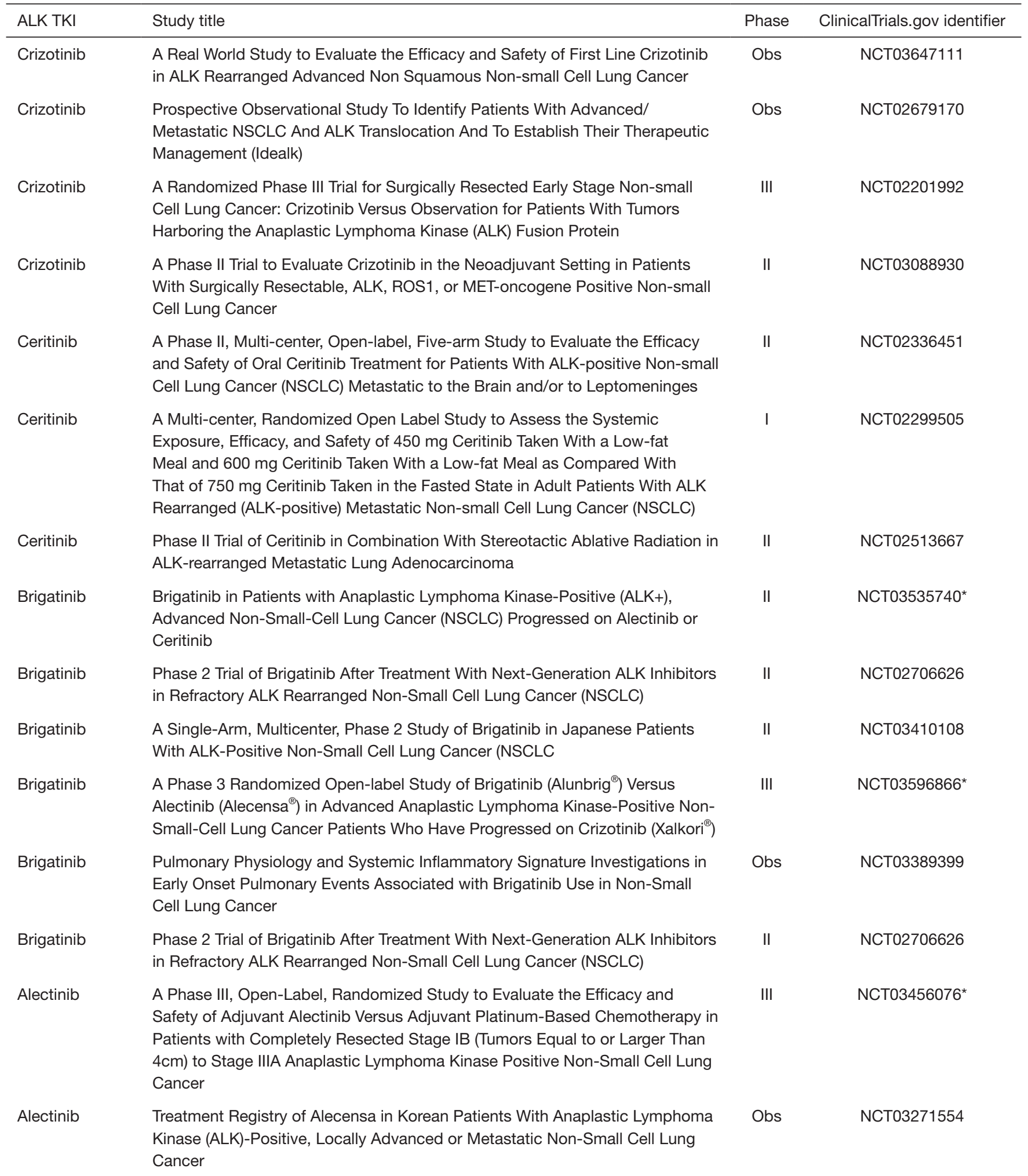

Table 1 (continued) 
Table 1 (continued)

\begin{tabular}{|c|c|c|c|}
\hline ALK TKI & Study title & Phase & ClinicalTrials.gov identifier \\
\hline Lorlatinib & $\begin{array}{l}\text { A Phase 3, Randomized, Open-label Study Of Lorlatinib (Pf-06463922) } \\
\text { Monotherapy Versus Crizotinib Monotherapy In The First-line Treatment Of } \\
\text { Patients With Advanced Alk-positive Non-small Cell Lung Cancer }\end{array}$ & III & NCT03052608 \\
\hline Lorlatinib & $\begin{array}{l}\text { A Phase 3, Randomized, Open-label Study of Lorlatinib (Pf-06463922) } \\
\text { Monotherapy Versus Crizotinib Monotherapy In The First-line Treatment Of } \\
\text { Patients With Advanced Alk-positive Non-small Cell Lung Cancer }\end{array}$ & III & NCT03052608 \\
\hline Ensartinib & $\begin{array}{l}\text { Phase } 3 \text { Randomized Study Comparing X-396 (Ensartinib) to Crizotinib in } \\
\text { Anaplastic Lymphoma Kinase (ALK) Positive Non-Small Cell Lung Cancer } \\
\text { (NSCLC) Patients }\end{array}$ & III & NCT02767804 \\
\hline
\end{tabular}

*, not yet recruiting. Obs, observational; TKI, tyrosine kinase inhibitor.

\section{New generation ALK inhibitors}

The first ALK inhibitor approved by FDA for refractory ALK-rearranged advanced NSCLC patients to a prior ALK TKI was ceritinib and, as evidenced by enzymatic essays, it is considered twenty times more effective compared to crizotinib (16). In ASCEND-1 phase I trial, 83 ALKrearranged NSCLC patients who became resistant to crizotinib were enrolled in the dose-escalation phases with ceritinib. Investigators described a 58\% ORR (95\% CI, 48-67\%) with a median PFS of 7 months (95\% CI, 5.6-9.5 months). Importantly, all 19 patients for whom tumor samples were available at crizotinib progression responded to ceritinib, independently of the acquired resistance mechanism. Considering these data, the study suggested that high responses were induced by ceritinib in those subjects who became refractory to crizotinib. However, in the subsequent evaluations, specifically in ASCEND-2 and ASCEND-5, respectively, despite similar numbers of patients undergone to ceritinib, the percentage of those heavily pre-treated (56\% versus $11 \%$, equal or more than three prior lines of treatment), a higher proportion of them with brain metastasis ( $71 \%$ versus $57 \%$ ), median PFS achieved with ceritinib calculated by independent review committee (IRC) was progressively shorter from ASCEND-1 to ASCEND-2 to ASCEND-5, dropping from 7.2 to 5.4 months (17). In the meantime, in both ASCEND-4 trial, which compared ceritinib to platinum/pemetrexed first-line chemotherapy and in ALEX one, which compared alectinib to crizotinib in patients with ALK-rearranged NSCLC, positive data emerged but median PFS in ceritinib study was 16.8 months versus alectinib one which reached 25.7 months, by IRC $(18,19)$.

Alectinib (RO5424802/CH5424802) is a secondgeneration, ATP-competitive ALK TKI, specifically designed to overcome crizotinib resistance. Unlike crizotinib, alectinib is not able to inhibit ROS1 or MET while it reduces RET activity with a comparable potency to that of ALK. In vitro alectinib has a 3-fold increase ALK inhibition compared to crizotinib (to date 53 and 150.8 $\mathrm{nM}$, respectively). Moreover in vitro alectinib is efficient in treating several crizotinib-resistant ALK mutations, such as C1156Y, F1174L, L1196M or R1275Q. In the same context, it also proved its efficacy against ALK-mutant ceritinib-resistant L1198F and moderate potency against composite mutations such as D1203N+F1174C (20).

As evidenced in vitro studies and unlike crizotinib and 
ceritinib, alectinib is not a substrate of the key efflux transporter, P-gp. This could clarify its higher concentration in cerebrospinal fluid (CSF) (close to $0.75 \mathrm{ng} / \mathrm{mL}$ ) compared to crizotinib $(0.616 \mathrm{ng} / \mathrm{mL}$, as reported by Costa et al.) supporting its suggested activity also in ALK-rearranged NSCLC patients with leptomeningeal disease (21). The phase III ALEX trial, comparing first-line alectinib to crizotinib in advanced ALK-rearranged NSCLC patients, confirmed a higher response rate $(82.9 \%$ versus $75.5 \%)$, a higher 12 -month event-free survival rate $(68.4 \%$ versus $48.7 \%$ ) with a lower probability of progressive disease ( $41 \%$ versus $68 \%$ ) and particularly CNS progression (12\% versus $45 \%$ ) with a lower rate of AEs ( $41 \%$ versus $50 \%$ ). Median PFS has not yet been reached with alectinib (95\% CI, 17.7 months to not estimated) and was 11.1 months with crizotinib (95\% CI, 9.1-13.1 months) (19).

Finally, if eventually a CNS progression arises under alectinib administration, Gainor et al. reported that dose escalation with alectinib ( $900 \mathrm{mg}$ twice daily) was able to reinduce CNS tumor response in two ALK-positive NSCLC patients who presented CNS relapse on alectinib standard dose (600 mg twice daily), suggesting that dosing strategies could eventually overcome reduced CNS activity (22).

Brigatinib (AP26113) was developed as a secondgeneration ALK TKI. It is able to inhibit both ALK and EGFR with also EGFR T790M and ALK L1196M mutants as showed in pre-clinical and first-in-human studies. Brigatinib was evaluated with two different regimens in a phase II trial including crizotinib-refractory ALK-rearranged NSCLC patients who were then randomized to oral brigatinib $90 \mathrm{mg}$ once daily (arm A) or brigatinib $180 \mathrm{mg}$ once daily ( $\mathrm{arm} \mathrm{B}$ ) with a 7-day lead-in at $90 \mathrm{mg}$. Median PFS as assessed by investigators was 9.2 and 12.9 months (95\% CI, 7.4-15.6 months and 95\% CI, 11.1 months to not reached) in arms A and B, respectively. ORR as assessed by investigators was $45 \%$ and $54 \%(97.5 \%$ CI, $34-56 \%$ and $97.5 \%$ CI, 43-65\%) in arms A and B, respectively (23). To date, in a recent press release of July 2018 from the global phase III ALTA-1L (ALK in lung cancer trial of AP26113 in 1st-line) randomized trial, investigators communicated that brigatinib too, at the first pre-specified interim analysis, reached its primary endpoint, evidencing a statistically significant advantage, compared to crizotinib, in PFS in naïve ALK-rearranged advanced NSCLC patients (24).

Finally, third-generation inhibitors are rising and lorlatinib (PF-06463922) as a reversible, potent ALK and ROS1 ATP-competitive inhibitor is one of them. In both vitro and xenograft models it demonstrated to be active in crizotinib-resistant cancers as well as against several resistant mutations in pre-clinical studies. In recently presented early phase II data a clinically meaningful activity was evidenced with substantial intracranial efficacy, particularly among patients harbouring ALK or ROS1 rearrangement and who were either treatment-naïve or failed a previous ALK inhibitor (25).

\section{Conclusions}

Considering previous data, the large number of ALK inhibitors is now a precious resource aiming to prolong PFS and finally OS, highlighting the perception that ALK-rearranged advanced NSCLC patients benefit from maintained ALK inhibition for as long as they possibly can. Integrated treatments for oligo-metastatic disease and CNS progression, beyond-progression approaches, rechallenges or optimal treatment sequences based on resistance mechanisms should be specifically defined for every single patient.

\section{Acknowledgements}

None.

\section{Footnote}

Conflicts of Interest: Prof. Silvia Novello declares speaker bureau relationship with Roche, MSD, BI, Takeda, Astra Zeneca, Pfizer, BMS. The other authors have no conflicts of interest to declare.

\section{References}

1. WHO Statistics. [Accessed on 21 August, 2018]. Available online: http://www.who.int/mediacentre/factsheets/ fs297/en/

2. Soda M, Choi YL, Enomoto M, et al. Identification of the transforming EML4-ALK fusion gene in non-small-cell lung cancer. Nature 2007;448:561-6.

3. Shaw AT, Yeap BY, Mino-Kenudson M, et al. Clinical features and outcome of patients with non-small-cell lung cancer whoharbor EML4-ALK. J Clin Oncol 2009;27:4247-53.

4. Sabir SR, Yeoh S, Jackson G, et al. EML4-ALK Variants: Biological and Molecular Properties, and the Implications for Patients. Cancers (Basel) 2017;9(9).

5. Lindeman NI, Cagle PT, Beasley MB, et al. Molecular 
testing guideline for selection of lung cancer patients for EGFR and ALK tyrosine kinase inhibitors; guideline from the College of American Pathologists, International Association for the Study of Lung Cancer, and Association for Molecular Pathology. J Thorac Oncol 2013;8:823-59.

6. Marchetti A, Di Lorito A, Pace MV, et al. ALK Protein Analysis by IHC Staining after Recent Regulatory Changes: A Comparison of Two Widely Used Approaches, Revision of the Literature, and a New Testing Algorithm. J Thorac Oncol 2016;11:487-95.

7. Hofman P. ALK in Non-Small Cell Lung Cancer (NSCLC) Pathobiology, Epidemiology, Detection from Tumor Tissue and Algorithm Diagnosis in a Daily Practice. Cancers (Basel) 2017;9(8).

8. Zhang X, Zhou JG, Wu HL, et al. Diagnostic accuracy of PCR for detecting ALK gene rearrangement in NSCLC patients: A systematic review and meta-analysis. Oncotarget 2017;8:75400-10.

9. Rozenblum AB, Ilouze M, Dudnik E et al. Clinical impact of hybrid capture-based next-generation sequencing on changes in treatment decisions in lung cancer. J Thorac Oncol 2017,12:258-68.

10. Solomon BJ, Mok T, Kim DW, et al. First-line crizotinib versus chemotherapy in ALK-positive lung cancer. N Engl J Med 2014;371:2167-77.

11. Lin JJ, Riely GJ, Shaw AT. Targeting ALK: Precision medicine takes on drug resistance. Cancer Discov 2017;7:137-55.

12. Doebele RC, Pilling AB, Aisner DL, et al. Mechanisms of resistance to crizotinib in patients with ALK gene rearranged nonsmallcell lung cancer. Clin Cancer Res 2012;18:1472-82.

13. Yoshida T, Oya Y, Tanaka K, et al. Differential crizotinib response duration among ALK fusion variants in ALKpositive non-small-cell lung cancer. J Clin Oncol 2016;34:3383-9.

14. Woo CG, Seo S, Kim SW, et al. Differential protein stability and clinical responses of EML4-ALK fusion variants to various ALK inhibitors in advanced ALKrearranged non-small cell lung cancer. Ann Oncol 2017;28:791-7.

15. Ziogas DC, Tsiara A, Tsironis G, et al. Treating ALK- positive non-small cell lung cancer. Ann Transl Med 2018;6:141.

16. Friboulet L, Li N, Katayama R, et al. The ALK inhibitor ceritinib overcomes crizotinib resistance in non-small cell lung cancer. Cancer Discov2014;4:662-73.

17. Wu F, Ou S. ASCEND-5: too little too late? J Thorac Dis 2017;9:3477-9.

18. Soria JC, Tan DSW, Chiari R, et al. First-line ceritinib versus platinum-based chemotherapy in advanced ALKrearranged non-small-cell lung cancer (ASCEND-4): a randomised, open-label, phase 3 study. Lancet 2017;389:917-29.

19. Peters S, Camidge DR, Shaw AT, et al. Alectinib versus Crizotinib in Untreated ALK-Positive Non-Small-Cell Lung Cancer. N Engl J Med 2017;377:829-38.

20. Gainor JF, Dardaei L, Yoda S, et al. Molecular Mechanisms of Resistance toFirst- and Second-Generation ALK Inhibitors in ALK -Rearranged Lung Cancer. Cancer Discov 2016;6:1118-33.

21. Costa DB, Kobayashi S, Pandya SS, et al. CSF concentration of the anaplastic lymphoma kinase inhibitor crizotinib. J Clin Oncol 2011;29:e443-5.

22. Gainor JF, Chi AS, Logan J, et al. Alectinib Dose Escalation Reinduces Central Nervous System Responses in Patients with Anaplastic Lymphoma Kinase-Positive Non-Small Cell Lung Cancer Relapsing on Standard Dose Alectinib. J Thorac Oncol 2016;11:256-60.

23. Wu J, Savooji J, Liu D. Second- and third-generation ALK inhibitors for non-small cell lung cancer. J Hematol Oncol 2016;9:19.

24. Takeda Announces Phase 3 Trial of ALUNBRIG® (brigatinib) Met Primary Endpoint Demonstrating Superiority in Progression-Free Survival Versus Crizotinib in Patients with ALK+ Advanced NSCLC Who are ALK Inhibitor Naïve. Available online: https://www.takeda.com/ newsroom/newsreleases/2018/takeda-announces-phase-3trial-of-alunbrig-brigatinib-met-primary-endpoint/

25. Solomon B, Shaw A, Ou S, et al. OA 05.06 Phase 2 Study of Lorlatinib in Patients with Advanced ALK+/ ROS1+ Non-Small-Cell Lung Cancer. J Thorac Oncol 2017;12:S1756.
Cite this article as: Vavalà T, Mariniello A, Novello S. Anaplastic lymphoma kinase tyrosine kinase inhibitors in nonsmall cell lung cancer. Transl Cancer Res 2019;8(Suppl 1):S48S54. doi: 10.21037/tcr.2018.10.23 